\title{
goG - die Neue Urbane Mobilität
}

Hans-Georg Höhne

Es wird ein umfassendes Verkehrskonzept für das urbane Umfeld beschrieben, welches durch den neuen kollaborativen Fahrzeugtyp mit dem Namen goG [go 'dschi] ermöglicht wird. Fahrzeuge dieses Typs können sich nahtlos im bestehenden Straßenverkehr eingliedern, ohne dass daran Anpassungen vorzunehmen sind. Auf Grund seiner kollaborativen Fähigkeiten können goGs aber auch zu Konvois des ÖPNVs formiert werden und annähernd $80 \%$ der im Vergleich für PKWs benötigten Parkplatzfläche einsparen. Desweiteren befähigen sie das goG in voller Fahrt von der Straße an eine über der Straße verlaufenden Umlaufseilbahn zu wechseln. Ein gittergleiches Netzwerk solcher Seilbahnen erlaubt das automatische Durchrouten von goGs 6 Meter über dem Straßenniveau, bei einer konstanten Geschwindigkeit von 50 km/h. An Seilbahnknotenpunkten werden durch Manipulatoren die goGs in voller Fahrt von einer Seilbahn in die vorgesehene nächste Seilbahn umgehängt. Durch hängebrückenartige Aufhängung der Umlaufseilbahnen oberhalb bestehender Straßenzüge werden keine zusätzlichen Flächen benötigt. Der Durchsatz einer Fahrbahn vergrößert sich durch die darüber geführte Umlaufseilbahn um mehr als das Doppelte. Ein autonomes Fahren bei maximal $6 \mathrm{~km} / \mathrm{h}$ zur nächsten goG-Linie des ÖPNV bzw. Seilbahn führt zu einer Vielzahl von Synergieeffekten.

Keywords: Seilbahn, Konvoi, Kollaboration, autonomes Fahren, individualisierter ÖPNV, Sicherheit

\section{Motivation für dieses neue Mobilitätskonzept goG}

\section{Bedarfe und Zielsetzungen}

Mobilität bedeutet im Kern eine Person von Ort A nach Ort B zu bringen.

Die Randbedingungen wie das geschehen kann und welcher Aufwand betrieben werden muss, hängt von der jeweiligen Mobilitätsform und dem Mix mit den anderen bestehenden Mobilitätsformen ab. Nur sehr bedingt lassen sich effiziente Schnittstellen zwischen den Mobilitätsformen untereinander realisieren.

Ohne darauf Rücksicht zu nehmen, werden hier die Bedarfe abstrakt aus den Perspektiven Anwender, Umwelt und Städteplaner formuliert, welche zugleich auch den Zielsetzungen des Mobilitätskonzeptes goG [go 'dschi] entsprechen.

- Anwender: Von A nach B kommen, zum Wunschzeitpunkt X - schnell, zuverlässig, barrierefrei, umstiegsfrei, umweltverträglich, kostengünstig, komfortabel, bei 
gleichzeitiger Wahrung der Privatsphäre, Schutz vor Witterung und Sicherheit vor Ansteckung mit Krankheiten, vor Belästigungen und Gewalt.

- Umwelt: emissionsfrei hinsichtlich Abgase (CO2, Feinstaub, Stickoxide), leise, ressourcenschonend in der Herstellung (Materialeinsatz) und im Betrieb (Energie und sonstige Betriebsmittel)

- Städteplaner: wenig Flächenbedarf, gute Integrationsfähigkeit in die bestehende Infrastruktur, Barrierefreiheit, deutliche Verbesserung der Leistungsfähigkeit des ÖPNV, preiswert, stufenweise implementierbar, für alle nutzbar (arm / reich, jung / alt, männlich / weiblich / sonstige, behindert / nicht behindert)

\section{Rekombination bewährter Konzepte, Neuinterpretation Konzepte, Sy- nergien aus anderen Bereichen}

- Rekombination bestehender Verkehrskonzepte: E-Bike, Rikscha, Seilbahn, Linienverkehr mit Bus und Bahn

- Neuinterpretation neuer, wenig verbreiteter Mobilitätskonzepte: Car-Sharing, Platooning, Uber, Autonomes Fahren, Flugtaxi, Luftbetankung

\section{Das Lösungskonzept goG - Beschreibung des Schwebe-/Fahrzeugs}

Das goG ist ein elektrisch angetriebenes Fahrzeug für zwei Personen. Mit seinem Aufhängebügel kann es sich direkt von der Straße in eine darüber verlaufende Umlaufseilbahn einklinken lassen. Die Reichweite an der Seilbahn ist unbegrenzt, da dort durch das Seil angetrieben.
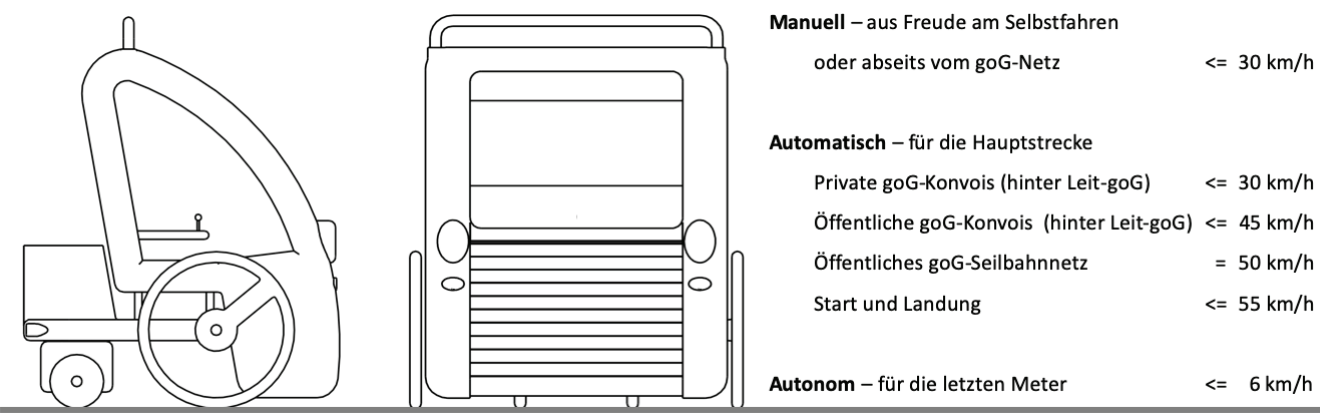

Abbildung 1: goG Seiten- und Frontansicht, sowie verfügbare Fahrmodi 
Mehrere goGs können kontaktlos zu einem Konvoi verbunden werden. Die goGs des Konvois folgen einfach automatisch dem jeweils voranfahrenden goG, der vorderste goG wird von einem Fahrer manuell gesteuert.

Abmessungen Länge $\times$ Breite $\times$ Höhe $=1,60 \mathrm{~m} \times 1,60 \mathrm{~m} \times 2,00 \mathrm{~m}$ inkl. Aufhängungskupplung. Der Ein- und Ausstieg erfolgt von vorne. Der Kofferraum ist hinten. Das goG kann sich auf der Stelle drehen, auf einer Fläche von $2 \mathrm{~m} \times 2 \mathrm{~m}$. Das maximal zulässige Gesamtgewicht beträgt $500 \mathrm{~kg}$. Die Mindestreichweite auf der Straße beträgt $20 \mathrm{~km}$. Die maximale Geschwindigkeit ist abhängig vom aktiven Fahrmodus

Durch den Fronteinstieg wird ein Maximum an Barrierefreiheit erreicht. Boden und Windschutzscheibe öffnen sich nach dem Rollladenprinzip nach unten und nach oben. Man stellt sich, auf der Straße stehend vor die Sitzbank, setzt sich hin und stellt seine Füße auf die Fußstütze. Dann schließen sich Windschutzscheibe und Boden und man befindet sich in einer rundum geschlossenen Kabine.

Es gibt kein Lenkrad. Eine manuelle Steuerung erfolgt über einen Joystick.

Um an den Kofferraum zu kommen geht man nicht um das Fahrzeug herum, sondern lässt es auf der Stelle um 180 Grad drehen.

\section{Integration von goGs in den urbanen Verkehr auf der Straße}

Der goG Linienverkehr ist die Adaption des bestehenden Konzeptes des öffentlichen Nahverkehrs. Es erfolgt dabei nur ein anderer Schnitt, der am Beispiel einer Buslinie erläutert wird.

Im Bus sitzen Fahrer und die Fahrgäste in einem Fahrzeug. Bei einem goG-Konvoi des ÖPNV teilt sich das was ein Bus darstellt auf in einen Leit-goG mit dem früheren Busfahrer und variabel vielen Leih-goGs bzw. Privat-goGs, welche sich an das Leit-goG hängen können und so einen Konvoi bilden. Die Straßenverkehrsordnung kennt bereits eindeutige Regeln für Konvois und damit auch für goG-Konvois.

Ein großer Unterschied zum als Beispiel genommenen Bus ergibt sich in der Durchführung des "Ein- und Ausstiegs" der Passagiere. Bei einer angenommenen Fahrt mit 10 Zwischenstationen hält der Bus zehnmal an, lässt Leute aus- und einsteigen, verkauft Tickets und fädelt sich wieder in den Straßenverkehr ein. Diese Vorgänge benötigen ein Vielfaches an Zeit im Vergleich zu der Zeit, die der Bus bräuchte, einfach an der Haltestelle vorbeizufahren. Und für einen Fahrgast ist dieser bei jeder Zwischenstation anfallende Zeitunterschied verlorene Zeit. 
Beim Linien-goG fährt der Konvoi an den Haltestellen ggf. etwas verlangsamt vorbei. Die dort auf die Linie wartenden goGs gliedern sich automatisch von der Haltebucht in die für sie freigemachte Lücken ein. Umgekehrt gliedern sich die dort "aussteigenden" goGs aus. Die Abstände zwischen den ausscherenden goGs und dem jeweiligen nachfolgenden goG sind entsprechend vergrößert, so dass die ausscherenden goGs automatisch abbremsen und in der Haltebucht anhalten können. Von dort aus kann man das goG verlassen und steht anderen Personen zur Nutzung zur Verfügung oder man fährt manuell gesteuert weiter.

Bei privaten goG-Konvois ist der Fahrer im Leit-goG kein Berufskraftfahrer wie beim ÖPNV, sondern eine Privatperson mit Führerschein. Auch ihm wird erlaubt sein ein oder mehrere goGs „anzuhängen“, um Personen und Güter transportieren zu können.

\section{Integration von goGs in den urbanen Verkehr über der Straße an Umlaufseilbahnen}

So wie es für Fahrzeuge vom Typ Bus, Tram exklusive Fahrtrassen geben kann und für S-Bahn, U-Bahn gibt, ist ein Kern des goG-Konzeptes, dass es für Fahrzeuge diesen Typs Schwebetrassen in Form von Umlaufseilbahnen geben kann. Besonders attraktiv hierfür ist der Luftraum über öffentliche Straßenflächen, da die darunter verlaufenden Straßen neben der bisherigen Funktion auch als Start- und Landebahn für den Übergang von goGs zwischen Straße und Seilbahn genutzt werden können. Beim Startvorgang wird das goG von einer an der Seilbahn befestigten Abhängung mit Greifer gegriffen und auf Flughöhe in 6 Metern oberhalb der Straße gezogen. Beim Landevorgang geschieht der Vorgang in umgekehrter Reihenfolge, indem die Abhängung das goG herablässt, auf dem Boden angekommen loslässt und den leeren Greifer zurück auf Flughöhe zieht. Durch diese Ausgestaltung des Überganges sind keinerlei Gebäudlichkeiten und damit verbundenen Zusatzflächen vorzusehen die einen Ein- und Ausstieg zur Seilbahn erfordern, da dieser einfach direkt auf der Straße passiert.

Eine Nutzung des Luftraums oberhalb öffentlicher Flächen mittels Umlaufseilbahnen erweitert zudem die Kapazität einer so überspannten Straße um die Förderkapazität der Seilbahn und hilft damit auch den Verkehr auf dem Boden zu entlasten. Seilbahnen haben die Eigenschaft, dass sie aufgrund des festen Abstandes zwischen den Abhängungen automatisch kollisionsfrei sind. Eine Luftüberwachung wie sie z.B. bei Flugtaxen erforderlich wäre, um diese Eigenschaft zu erreichen, entfällt konstruktionsbedingt. Ebenso entfällt bei einem goG der Energieaufwand von Fluggeräten, um sich überhaupt in der Luft zu halten, sowie die Luft- und Schmutzverwirbelungen, da mit Hilfe der Seilbahnen die Luft für goGs Haken bekommt. 
Die Umlaufseilbahnen bilden ein Netzwerk. Sie sind kreuzungsfrei, indem sich an Kreuzungen und Gabelungen verschiedene Seilbahnen treffen und dort auf Flughöhe installierte Manipulatoren goGs in voller Fahrt, von der einen zur nächsten Seilbahn umhängen.

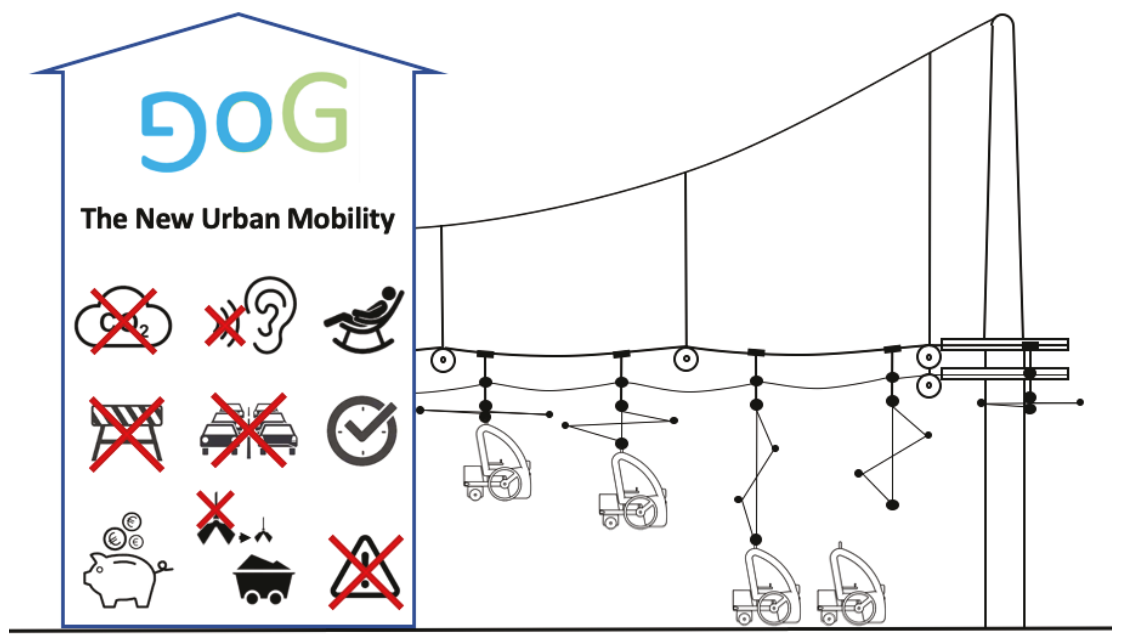

Abbildung 2: Ablauf goG Wechsel zwischen Seilbahn und Straße

Die Seilbahnen bewegen sich synchron mit 50,4 km/h was $14 \mathrm{~m} / \mathrm{s}$ entspricht. Bei einem angenommenen Abstand von $14 \mathrm{~m}$ zwischen den Abhängungen entspricht das also einer möglichen Transportkapazität von 1 goG/s für eine Richtung. Bezogen auf eine Stunde sind das maximal 3600 goGs mit maximal 7200 Personen pro Richtung. Für den maximalen Durchsatz von Fahrzeugen auf einer einspurigen Straße können 2500 Autos/h angesetzt werden (Randelhoff, Martin: 2011). Alles darüber hinaus führt zum Einbruch des Durchsatzes auf Grund eines dann entstehenden stockenden Verkehrs oder Staus. Das entspricht einer Kapazitätserhöhung einer einspurigen Straße von maximal 2500 Fahrzeugen ohne Seilbahn auf 6100 Fahrzeuge mit Seilbahn. Das ist deutlich mehr als eine Verdopplung der Straßenkapazität! Staus sind bei goGs-Seilbahnen prinzipbedingt ausgeschlossen.

Mit dem Einchecken eines goGs ins Seilbahnnetz sind alle erforderlichen Abhängungen auf allen Seilbahnteilstrecken der "Flugroute" reserviert. Im Seilbahnnetz besteht im Gegensatz zum Straßennetz auch eine konstante Reisegeschwindigkeit von 50,4 km/h. Die Ankunftszeit ist bei Fahrt am Seil im Gegensatz zur Fahrt auf der Straße verlässlich planbar. 


\section{Integration von goGs im ruhenden Verkehr}

Parkflächen für PKWs gibt es am Straßenrand, in Parkhäuser, bei Supermärkten, in Garagen und Tiefgaragen. goGs können diese gleichermaßen nutzen, aber es gibt bessere Lösungen, wenn man den Formfaktor und die Kollaborationsfähigkeit von goGs ausnutzt.

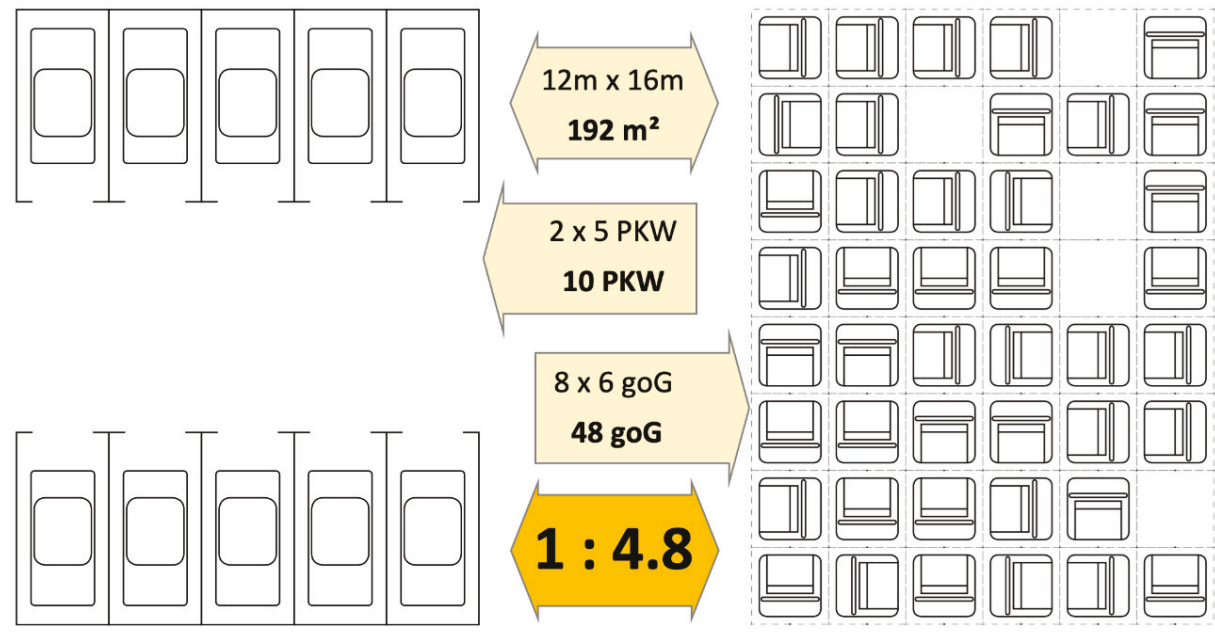

Abbildung 3: Vergleich Flächenverbrauch klassischer Parkplatz mit goG-Parkplatz

Ein reiner goG-Parkplatz ist deutlich platzsparender, da hier die smarten Eigenschaften von goGs ausgenutzt werden. Ähnlich wie man sich bei einem Rockkonzert durch die dichte Menge schiebt und die Menschen um einen herum durchlassen, so lassen goGs einen zur Ausfahrt strebenden goG auch durch.

Im voranstehenden Bild sind 10 Parkplätze nach klassischer Anordnung (VBG-Hamburg: 2013) und 48 Parkplätze nach goG-Anordnung auf gleicher Fläche dargestellt. Dabei können mit goGs 4,8-mal so viele Fahrzeuge auf der Parkfläche von 10 Autos untergebracht werden. Im Gegensatz zum klassischen Parkplatz, wo es nur zwei Möglichkeiten zur Ein- und Ausfahrt gibt, können beim goG-Parkplatz potentiell alle Außenkanten als Ein- und Ausfahrt verwendet werden.

Dieses smarte Verhalten führt zu mehreren Vorteilen im täglichen Umgang. Beim Supermarkt fährt einen das goG wie bei einer Hotelvorfahrt direkt vor die Tür des Supermarktes und lässt einen aussteigen. Das goG parkt selbstständig in der beschriebenen 
Art und Weise. Man kauft ein, beim Passieren der Kasse wird das goG automatisch gerufen und man wird vom goG wieder direkt vor der Türe abgeholt. Man lädt seine Einkäufe ein, steigt ein und fährt weg.

Was ist daran anders als beim Einkaufen mit dem Auto?

Man braucht keinen Parkplatz suchen, nicht einparken, keinen Einkaufswagen suchen, diesen abholen, dann zur Tür über den Parkplatz des Supermarktes laufen und dem Risiko ausgesetzt zu sein mit Autos zu kollidieren.

Ein goG-Parkplatz braucht keinen Platz für Wege zu den einzelnen Parkplätzen, was den erforderlichen Flächenverbrauch deutlich senkt. Es gibt auch keinen Verschnitt pro Parkplatz, da goGs alle gleich groß sind. Es braucht auch keinen extra Abstand zwischen den Fahrzeugen, um seitliche Einstiegstüren öffnen zu können. Jeder goG braucht für sich einfach nur eine Fläche von $2 \times 2$ Metern und es müssen nur wenige Flächen dieser Größe frei bleiben, damit sich goGs "durchschlängeln" können.

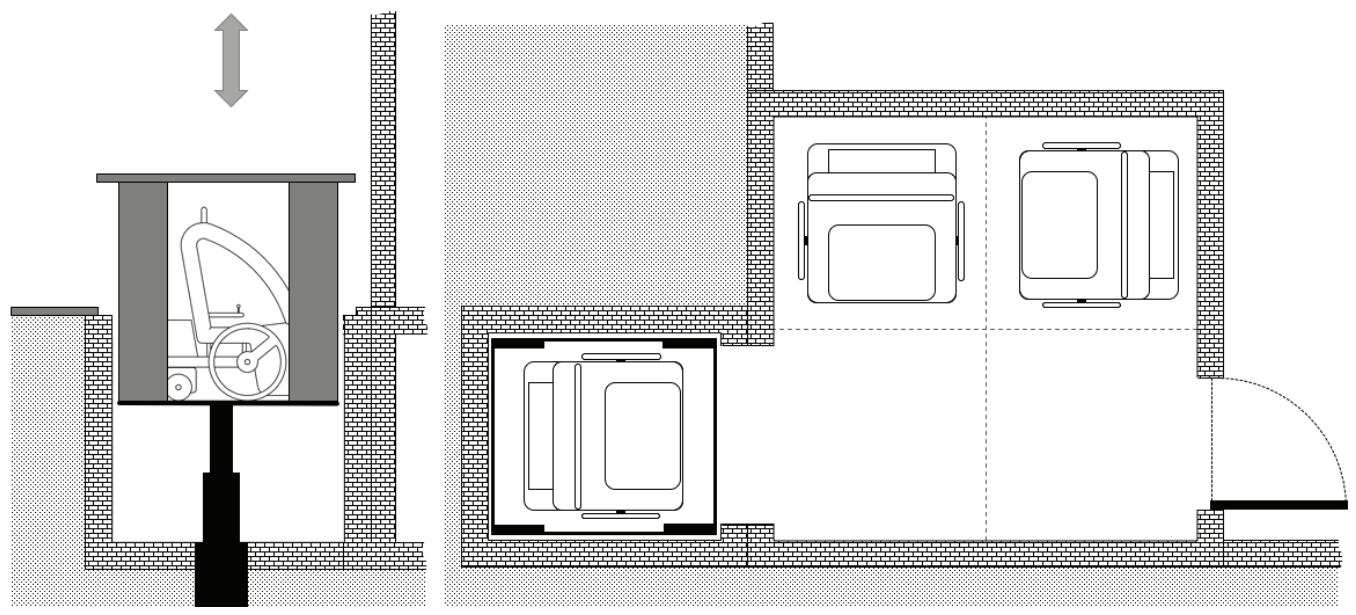

Abbildung 4: goG Kellerstellplatz mit Aufzug

Ein goG-Parkplatz im Wohnhauskeller ist eine elegante Variante auch bei bestehenden Wohnhäusern aus einem normalen Kellerraum eine Multi-goG-Garage zu machen. Einzige Voraussetzung hierfür ist eine $2 \times 2 \mathrm{~m}$ große Fläche direkt an der Hauswand, welche durch goGs angefahren werden kann. Im Idealfall ist diese direkt vor dem Eingang des Hauses. Dort ist ein kleiner, z. B. hydraulischer Aufzug eingelassen, welcher einen goG vom Kellerniveau auf das Straßenniveau heben, bzw. umgekehrt absenken kann. Bei der oberen Stellung ist das Dach des Aufzuges Vordach des Einganges und der 
Aufzugboden Boden des Durchgangs zur Haustüre. In der unteren Stellung ist das Dach des Aufzugs der Boden des Durchgangs zur Haustüre. Die Kellerwand zwischen Aufzugsschacht und Kellerraum erfordert einen Durchbruch von 1,80 × 2,10 m. Der Kellerraum benötigt pro goG eine Fläche von $2 \times 2$ Metern und eine freie Fläche dieser Größe zum Rangieren. Im obigen Bild ist die Konstellation mit 3 goG-Stellplätzen in einem $16 \mathrm{~m}^{2}$ großen Raum dargestellt. Eine installierte Ladestation wird automatisch nacheinander von den abgestellten goGs angefahren, um sich daran automatisch aufzuladen. Man kann im Keller das goG be- und entladen, ein- und aussteigen und fährt über den Aufzug im goG sitzenden zwischen Straßen- und Kellerniveau hoch und run-

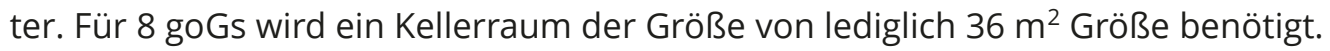

\section{Schnittstellen zwischen Land und Stadt}

Für die Menschen, die im Umland leben aber in der Stadt arbeiten, gibt es aktuell im Wesentlichen drei Alternativen: Sie pendeln mit öffentlichen Verkehrsmitteln, mit inrem Auto oder einer Kombination aus beidem.

Park and Ride ist das bestehende Konzept für die Kombination aus beidem. Mit dem eigenen Auto die Strecke zwischen Wohnort und Stadtrand und die zwischen Stadtrand und Arbeitsplatz mit den öffentlichen Verkehrsmitteln. goGs machen dieses Konzept attraktiver, denn Leute, die von außen mit ihrem Auto in die Stadt pendeln, steigen auf dem Park and Ride Parkplatz direkt in einen neben ihrem Auto stehenden goG um und fahren nahtlos, ohne Laufwege, ohne Pufferzeiten, ohne Wege- und Wartezeiten, ohne der Witterung ausgesetzt zu sein weiter. goGs stehen hierzu an der Einfahrt von Park und Ride Parkplätzen. Wenn ein Auto einfährt folgt ein goG dem Auto oder noch besser, fährt dem Auto voraus und führt es so zu einer freien Parklücke.

Aber auch der Übergang von der Bahn auf goGs „Rail and goG" funktioniert nach dem bewährten Prinzip des klassischen Taxi-Standes vor dem Bahnhof. Ganz analog dazu gibt es einen goG-Stand an dem man zwischen goG und Bahn wechseln kann.

\section{Schnittstelle zwischen Stadt und Land}

Für Menschen, die in der Stadt mit einem gut funktionierenden öffentlichen Nahverkehr leben, gibt es wenig Anreize ein eigenes Auto zu besitzen, da es nur selten genutzt wird. Allein das Thema Parkplatzsuche schreckt ab das eigene Auto zu nehmen, wenn es nicht unbedingt nötig ist. Für Fahrten über das Land ist das Auto das mit Abstand flexibelste Verkehrsmittel. Aber hierzu muss man ja nicht zwingend selbst ein Auto 
besitzen und falls doch, muss das Auto trotzdem nicht zwingend direkt vor der Haustüre im dicht besiedelten Stadtgebiet parken.

Die Idee ist hier "Ride and Drive“. Man fährt also mit dem goG zum Stadtrand direkt zu einem gemieteten Auto oder dem eigenen dort abgestellten Privatwagen. Man braucht dann nur vom goG in das direkt danebenstehende Fahrzeug umzusteigen.

\section{Schutz und Sicherheit}

\section{Schutz vor Witterung und Schutz der Privatsphäre vor Belästigung, Diebstahl und Überfällen}

Mit dem goG fährt man in einer für den Transfer von Ort A nach Ort B exklusiv genutzten Kapsel. Diese schützt nicht nur vor Witterungseinflüsse wie Sonne, Wind, Regen, Hitze, Kälte, Schnee und Eis, sondern auch vor Belästigungen, Taschendiebstählen und Überfällen. Belästigungen können im ÖPNV viele Formen annehmen, wie z.B. Anmache, Geruchs-, Geräuschbelästigung oder Körperkontakt.

\section{Sicherheit vor Infektionsübertragungen}

Es gibt wie beim Auto auch, beim Privat-goG keine Infektionsgefahr auf Grund von Kontakten mit Fremden, da man in seinem individuellen Fahrgastraum unterwegs ist. Bei Leih-goGs ist es leicht möglich eine vollständige Innenraumdesinfektion vor jedem Passagierwechsel vorzunehmen, z. B. durch eine kurze und intensive Bestrahlung des Innenraumes mit UV-Licht.

\section{Schutz der Umwelt}

Der Schutz der unmittelbaren Umwelt erfolgt durch emissionsfreien Antrieb des goG selbst, als auch der emissionsfreie Antrieb der Seilbahnen.

Im Vergleich zu einem PKW werden deutlich weniger Ressourcen zur Herstellung eines goGs benötigt, bei gleicher Transportkapazität von durchschnittlich 1,5 Personen pro Fahrzeug (FIS Mobilität und Verkehr:2019).

Die Herstellung von goGs und deren Komponenten sollte CO2-neutral erfolgen, ebenso die Erzeugung der Antriebsenergie. Durch die flächige Verteilung des Seilbahnnetzes kann auch direkt vor Ort auf Hausdächern installierte Photovoltaik zum Antrieb der Umlaufseilbahnen verwendet werden.

Die Versiegelungsfläche durch Seilbahnen ist vernachlässigbar. Die Versieglungsfläche durch Parkplätze reduziert sich um fast 80 Prozent im Vergleich zum PKW auf Grund 
der Größe und den Kollaborationseigenschaften des goGs. Weitere positive Umwelteffekte ergeben sich durch die optimale Verteilungsmöglichkeit auf Grund des autonomen Fahrens, welche einen reduzierten Fahrzeugbestand ermöglichen und die damit einhergehenden Einsparungen an Ressourcen.

Reduzierte Parkplatz- und Verkehrsflächen lassen deren Entsiegelung und Renaturierung zu. Dies erhöht insbesondere die Lebensqualität der dortigen Anwohner.

\section{Sicheres und ethisch unbedenkliches Autonomes Fahren mit 6 km/h}

Das goG bleibt bei externer Berührung sofort stehen. Der Bremsweg ist dabei mit höchstens $20 \mathrm{~cm}$ so kurz, dass eine angefahrene Person höchstens einen leichten Schupser erfährt und im schlimmsten Fall stürzt. Um das goG wieder weiterfahren zu lassen, braucht nur eine natürliche Person z.B. sagen "goG, du darfst weiterfahren", um es wieder zu entsperren.

Unter diesem Hintergrund entfällt die Beantwortung ethischer Fragen, wie "Fahre ich in die Schülergruppe oder in die Rentnergruppe?" durch den Autopiloten, da erstens ein goG keine Fläche befährt, wenn diese belegt ist, egal ob mit Rentnern, Schülern oder sonstigen Objekten und zweitens es wie zuvor beschrieben sofort stoppt, falls es zu einem direkten Kontakt gekommen ist

\section{Literaturverzeichnis}

Randelhoff, Martin (2011): Die drei Haupttheoreme der Stauforschung: Der Schmetterlingseffekt, unsichtbare Wellen (= Phantomstau) und die Tragik des Zufalls. https://www.zukunft-mobilitaet.net/3344/analyse/wie-entstehen-staus-phantomstau, abgerufen am 07.03.2021.

VBG-Hamburg (2013): PKW-Stellplatz-Flächen. https://www.zukunft-mobilitaet.net/3344/analyse/wie-entstehen-stausphantomstau, abgerufen am 07.03.2021.

FIS Mobilität und Verkehr (2019): PKW-Besetzungsgrad bei der privaten Autonutzung. https://www.forschungsinformationssystem.de/servlet/is/79638/, abgerufen am 07.03.2021.

\section{Kontakt}

Dipl.-Inform. Hans-Georg Höhne

Untere Krebsbachstr. 11

87752 Holzgünz

hans-georg.hoehne@t-online.de 